\title{
Design of high electron mobility devices with composite nitride channels
}

\author{
Madhusudan Singh ${ }^{\text {a) }}$ and Jasprit Singh \\ Department of Electrical Engineering and Computer Science, University of Michigan, Ann Arbor, \\ Michigan 48109
}

(Received 24 February 2003; accepted 3 June 2003)

\begin{abstract}
Field effect transistors based on a GaN/AlGaN system have shown remarkable performance characteristics in a wide range of device applications. However, due to the large effective mass of $\mathrm{GaN}$, the mobility in the channel is small. In this work, we consider a GaN/AlGaN structure with a thin InN channel of the order of a few monolayers. We find that mobility in the channel can improve considerably while breakdown characteristics are not expected to suffer. Mobilities of $\simeq 2500 \mathrm{~cm}^{2}(\mathrm{~V} \mathrm{~s})^{-1}$ are predicted along with high sheet charges for low interface disorder. Good agreement with experimental results is observed for higher degrees of disorder within the model. At higher electric fields, we find that most electron population transfers to higher valleys or other subbands that lie in AlGaN or GaN. We also compare the low-field mobility-charge product for this structure with the conventional $\mathrm{AlGaN} / \mathrm{GaN}$ structure and find that the two values are similar.

(C) 2003 American Institute of Physics. [DOI: 10.1063/1.1594272]
\end{abstract}

\section{INTRODUCTION}

In electronic devices such as field effect transistors (FETs), there are regions (under device operating conditions) where electric fields are small (for instance, in the sourcegate region) and other regions where electric fields are very high (such as the gate-drain region). For optimum performance, one would need a high mobility (low effective mass and low band gap) material for low field regions and a high saturation velocity and high breakdown field material for the high field regions. In this article, we examine a GaN/AlGaN HFET with a very thin $\mathrm{InN}$ intermediate region $(\sim 15 \AA)$. The motivation for the study is the expectation that at low fields, the electron distribution will be in the $\mathrm{InN}$ region and at high fields, there will be essentially no overlap of the electronic state with the $\mathrm{InN}$ region. Thus we can expect high mobility and high saturation velocity without significant increase in breakdown. The use of the InN channel is motivated by recent reports ${ }^{1-3}$ that $\mathrm{InN}$ has a smaller band gap than previously thought ${ }^{4,5}(0.9 \mathrm{eV}$ versus the previous 1.89 $\mathrm{eV}$ ) and thus may have a very small effective mass. ${ }^{6-8}$ There are currently no velocity-field calculations for a GaN/InN/ AlGaN composite structure. Previous work in design of high electron mobility transistors (HEMTs) has addressed this problem for bulk nitride semiconductors ${ }^{9}$ or the problem of two-dimensional transport in GaN channels, with fairly high peak electron velocities. ${ }^{10}$ Therefore it is not known if there are any benefits in using the newer structures. We report a comparative study on $\mathrm{GaN} / \mathrm{Al}_{0.37} \mathrm{Ga}_{0.63} \mathrm{~N}$ and $\mathrm{GaN} / \mathrm{InN} / \mathrm{Al}_{0.37} \mathrm{Ga}_{0.63} \mathrm{~N}$.

Figure 1 is a schematic for one such structure. It employs a high band gap material (in the present work, $\left.\mathrm{Al}_{0.37} \mathrm{Ga}_{0.63} \mathrm{~N}\right)$ for the gate $(\mathrm{G})$, which reduces the probability of tunneling, heavily doped drain (D) and source (S) regions which facilitate conduction from the two contacts. A low

${ }^{a)}$ Electronic mail: chhabra@eecs.umich.edu band gap material (in the present work, InN) is used in the channel region, while another high band gap material (in the present work, GaN) is used in the bulk region. Owing to piezoelectric effect and spontaneous polarization, ${ }^{11-14}$ a sheet charge of $\sim 10^{14} \mathrm{~cm}^{-2}$ can be induced at the heterostructure interface between the gate and the channel material. This forms the two-dimensional electron gas (2DEG) in this structure and supports conduction.

All materials in the structure are taken to be intentionally undoped. Previous studies ${ }^{15}$ have indicated that even for impurity concentrations as high as $5 \times 10^{17} \mathrm{~cm}^{-3}$ in a low band gap material like GaAs, the scattering rates are $\leqslant 10^{11} \mathrm{~s}^{-1}$. In our study, these scattering rates are lower than even the weakest scattering mechanism (see Fig. 2). Experimental studies in nitrides grown by molecular beam epitaxy (MBE) also discard ionized impurity scattering in favor of alloy scattering and interface roughness scattering. ${ }^{16}$ Hence we neglect ionized impurity scattering in our study.

\section{FORMALISM}

The simulation of the structure involves the selfconsistent solution of Poisson and Schrödinger equations in one dimension and subsequent use of the solution in a Monte Carlo simulation.

\section{A. Charge control}

Owing to piezoelectric effect and spontaneous polarization for $\mathrm{AlGaN}$ and $\mathrm{InN}$ grown on $c$-axis $\mathrm{GaN}$, there is a large charge density induced at the interfaces of different materials. Using parameters ${ }^{12}$ available in literature, we find that the fixed charges at the interfaces (for structures studied in this work, see Sec. III) are 

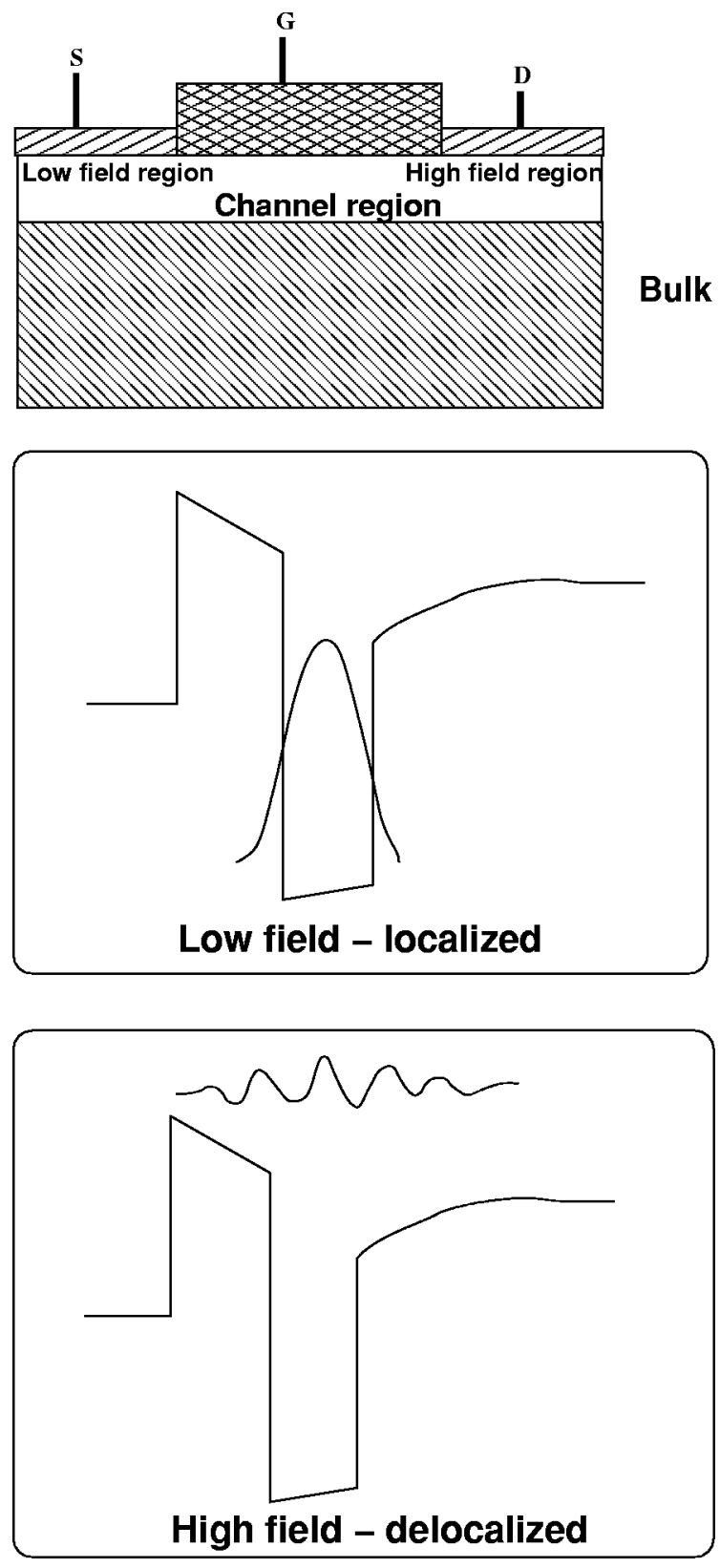

FIG. 1. A schematic showing a composite nitride channel device. The channel region is composed of a low band gap material such as InN. The thicknesses of various layers are chosen to provide wave function switching at a reasonably high electric field.

$$
\begin{aligned}
& \text { At the GaN/InN interface: }-1.65 \times 10^{14} \mathrm{~cm}^{-2} \text {. } \\
& \text { At the InN/Al }{ }_{0.37} \mathrm{Ga}_{0.63} \mathrm{~N} \text { interface: } 1.86 \\
& \times 10^{14} \mathrm{~cm}^{-2} \text {. }
\end{aligned}
$$

These values of fixed charges are used in the charge control calculation. The details of the charge control calculation have been dealt with extensively in the past. ${ }^{13,14}$ This essentially involves the solution of the Schrödinger [Eq. (1)] and Poisson [Eq. (2)] equations (in one-dimensional) selfconsistently to obtain the potential profile, carrier wave functions, and energy levels.

$$
-\frac{\hbar^{2}}{2 m^{*}} \frac{d^{2} \psi_{n}(z)}{d z^{2}}+V(z) \psi_{n}(z)=E_{n} \psi_{n}(z)
$$

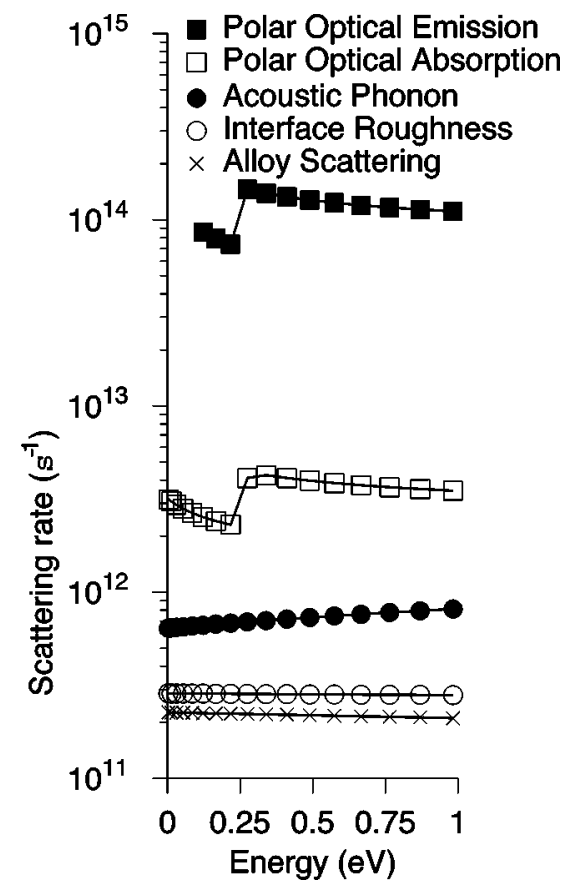

FIG. 2. Interface roughness, alloy scattering, acoustic phonon, polar optical phonon absorption, and polar optical phonon emission scattering rates for the $\mathrm{GaN} / \mathrm{InN} / \mathrm{Al}_{0.37} \mathrm{Ga}_{0.63} \mathrm{~N}$ structure at $300 \mathrm{~K}$. Polar optical phonon emission dominates, being more than an order of magnitude higher than the other rates. Polar optical phonon emission is zero below the threshold energy, hence those points do not show on this plot. Interface roughness parameters: $L=5 \AA$ and $\Delta=5 \AA$. Alloy scattering parameter: $R=5 \AA$.

$$
\text { and } \frac{d^{2} V(z)}{d z^{2}}=-\rho
$$

The Schrödinger equation yields the confined charge terms in the Poisson equation, which in turn determines the potential profile. This potential profile is fed back into the Schrödinger equation and this process is repeated until the solution of the Poisson equation goes to convergence.

The resultant wave functions from the solution of Eqs. (1) and (2) are then used to calculate overlap integrals, $I_{m n}$, which are used in the calculation of the intersubband matrix elements [Eq. (4)].

$$
I_{m n}=\int_{\text {entire device }} \psi_{m}^{*}(z) \psi_{n}(z) d z
$$

It must be noted that the above wave functions are normalized over the entire device.

\section{B. Monte Carlo simulation}

Transport properties are calculated by the usual Monte Carlo method. ${ }^{10,15,17,18}$ This involves simulation of the motion of a single electron over a long period of time (to obtain carrier velocities) or simulation of many carriers over a short period of time to obtain the distribution of velocities. This Monte Carlo process models the dominant scattering mechanisms at $300 \mathrm{~K}$-acoustic and polar optical phonon emission and absorption, interface roughness and alloy scattering, in- 
TABLE I. Parameters used in Monte Carlo simulation.

\begin{tabular}{cccc}
\hline \hline \multicolumn{4}{c}{ Monte Carlo parameters } \\
\hline $\begin{array}{c}\text { Number of } \\
\text { electrons }\end{array}$ & $\begin{array}{c}\text { Initial } \\
\text { energy }(\mathrm{eV})\end{array}$ & $\begin{array}{c}\text { Simulation } \\
\text { time }(\mathrm{ns})\end{array}$ & $\Gamma\left(\mathrm{s}^{-1}\right)$ \\
\hline 1 & 0.026 & 16 & $6.16 \times 10^{14}$ \\
\hline \hline
\end{tabular}

terspersed with periods of free flight. The process proceeds in the following steps (for the GaN/AlGaN and GaN/InN/ AlGaN structures):

(1) Initial calculation of the scattering rates for intra- and inter-subband scattering.

(2) Injection of electrons into the structure. Carriers are initially injected with thermal velocity.

(3) Random choice of scattering event as one of acoustic phonon scattering, polar optical phonon absorption and emission, intervalley scattering, interface roughness scattering, alloy scattering, etc.

(4) Updating the final position and velocity of the electron after the scattering event.

(5) Free flight.

Steps (3)-(5) are repeated until the end of the simulation. For the purpose of the simulation, we follow the motion of one electron, for a long period of time (in the present case, $16 \mathrm{~ns}$ ). This long period of simulation is chosen to ensure that the velocity at the end of the simulation is a steady state velocity (corresponding to saturation), uniquely corresponding to the electric field in the channel. The resultant curve is used to deduce the low field drift mobility, $\mu_{l}$. The advantage of using this method is readily apparent —one can obtain a very fine level of detail about electron transport. The parameters ${ }^{8}$ used in the Monte Carlo process are summarized in Table I.

According to first order perturbation theory, the scattering rate per unit time into any final state is given by the Fermi golden rule, ${ }^{19,20}$

$$
\begin{aligned}
& M_{m k}=\left\langle k\left|H_{\text {pert }}\right| m\right\rangle, \\
& W_{m}=\frac{2 \pi}{\hbar} \sum_{k} \delta\left(E_{m}-E_{k}\right)\left|M_{m k}\right|^{2},
\end{aligned}
$$

where $H_{\text {pert }}$ is the perturbation Hamiltonian for the interaction, and $k$ is the index over all final states.

The details of calculation in quasi-two-dimensional systems are slightly different from what Eq. (5) would suggest. ${ }^{15}$ The matrix element used in Eq. (5) is calculated by integrating an overlap integral term [either similar to Eq. (3) or similar to it] with the three-dimensional matrix element. In particular, for phonon scattering, we have

$$
\begin{aligned}
& I_{m n}(q)=\int_{\text {entire device }} \psi_{m}^{*}(z) \psi_{n}(z) d z, \\
& \left|M_{m m}\right|^{2}=\int|M(Q, q)|^{2}\left|I_{m n}(q)\right|^{2} d q,
\end{aligned}
$$

(a)

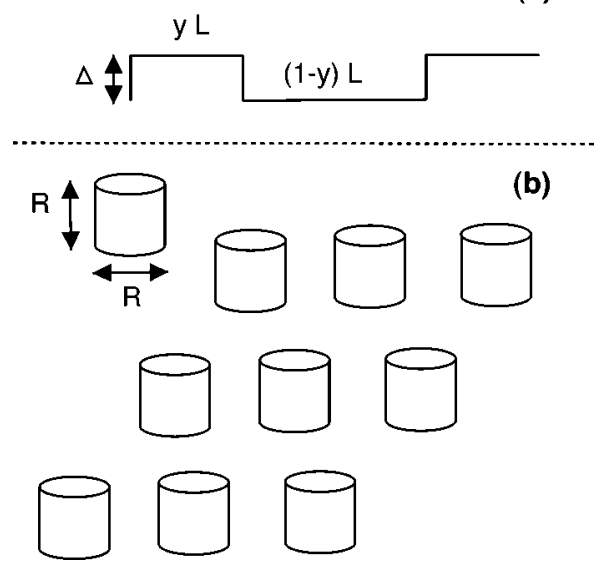

FIG. 3. Schematic for (a) interface roughness and (b) alloy scattering. For alloy scattering, an array of cylindrical scattering centers is used, with such arrays arranged randomly throughout the bulk of the alloyed layer.

where $Q$ and $q$ are components of phonon wave vectors parallel and perpendicular to the heterointerface, respectively.

We have also included the effect of interface roughness scattering (see Appendix A for details). Though prior studies exist for this scattering mechanism, ${ }^{21}$ they do not include the details of energy quantization explicitly. It is customary to use an energy independent expression for alloy scattering. ${ }^{8,22}$ However, we use an energy dependent expression (see Appendix B) for completeness. The geometry used in these calculations is shown in Fig. 3.

\section{Material parameters}

The values of different material constants have been taken from currently available literature. ${ }^{12,23-27}$ There is considerable uncertainty about values of nonparabolicity of upper valleys in the conduction band of wurtzite nitrides. ${ }^{28} \mathrm{We}$ use values of nonparabolicities obtained from a first principles calculation. $^{29}$

The relation of effective mass of an electron in a semiconductor to the band gap is described quite accurately by the $\mathbf{k} \cdot \mathbf{p}$ formalism: ${ }^{6-8}$

$$
\frac{1}{m^{*}}=\frac{1}{m_{0}}+\frac{2 p_{c v}^{2}}{3 m_{0}^{2}}\left(\frac{2}{E_{g \Gamma}}+\frac{1}{E_{g \Gamma}+\Delta}\right),
$$

where $\Delta$ is the separation between the heavy-hole and splitoff bands.

The fundamental band gap of InN was long thought of being around $1.89 \mathrm{eV}^{4,5}$ However, recent work indicates that the actual band gap of $\mathrm{InN}$ is much smaller, about $0.9 \mathrm{eV}{ }^{1-3}$ Using parameters in literature, ${ }^{30}$ we obtain a newer value of the effective mass $\left(\approx 0.05 m_{0}\right)$. These values are then used in our calculations.

\section{Parameter averaging}

It must be noted that in the Monte Carlo approach, the electron is treated as a point particle along the channel plane, but in the growth direction ( $z$ direction), it is represented by an envelope function, calculated earlier in Sec. II A. The envelope function for the ground state is localized very close to 
GaN/AlGaN or InN/AlGaN interfaces (as the case may be). For higher subbands, it is spread into the $\mathrm{GaN}$ or $\mathrm{AlGaN}$ region. To calculate the scattering rates for a state that overlaps several different regions, we need to use a reasonable averaging procedure.

We average any given material parameter over the number of layers, $N$, located at $z_{i}, i=1, \ldots, N$. Thus each subband has its own value of the relevant parameter. We take the following definition for the subband dependent value of a material parameter, $G_{j}$, since the wave functions are normalized over the entire device,

$$
\begin{aligned}
f_{j i} & =\int_{z_{j-1}}^{z_{j}} \psi_{i}^{*}(z) \psi_{i}(z) d z, \\
G_{j} & =\sum_{i=1}^{N} f_{j i} G_{i} .
\end{aligned}
$$

Equation (8) is used for all parameters, except effective masses, which are averaged harmonically,

$$
\frac{1}{m_{j}^{*}}=\sum_{i=1}^{N} \frac{f_{j i}}{m_{i}^{*}} \text {. }
$$

In Eqs. (8) and (9), the value of $G_{i}$ and $m_{i}^{*}$ on the right-hand side is the material value in each layer. These space-averaged subband dependent parameters are then used in all the calculations in the simulation.

\section{RESULTS}

Simulations were carried out for the following structures: $\quad \mathrm{GaN} / \mathrm{Al}_{0.37} \mathrm{Ga}_{0.63} \mathrm{~N} \quad(80 \quad \AA) \quad$ and $\mathrm{GaN} / \mathrm{InN}(15 \AA) / \mathrm{Al}_{0.37} \mathrm{Ga}_{0.63} \mathrm{~N}(80 \AA)$.

The reason for the choice of layer thicknesses as above is explained in Sec. III A. Further, the scattering rates corresponding to phonon scattering, considered in this study, were calculated at $300 \mathrm{~K}$. The results of that calculation are shown in Fig. 5. In all the simulations, the interface roughness parameter $y$ was chosen to be 0.5 (i.e., half of the interface between two materials is perturbed [see Fig. 2(a)]).

\section{A. Charge distribution}

In Fig. 4 we present results for the charge control study of the structure: $\mathrm{GaN} / \mathrm{InN} / \mathrm{Al}_{0.37} \mathrm{Ga}_{0.63} \mathrm{~N}(80 \AA)$. It may be noted that the charge induced in the channel region $(\mathrm{InN})$ layer increases as the thickness of the channel layer is increased. This is due to better confinement of the ground state wave function in the lower band gap material. However, there is an additional constraint - the overall strain energy of the structure. Experimentally, ${ }^{31}$ it has been found that growth of pure InN layers of thickness as high as $10-20 \AA$ is possible. However, structures with lower thicknesses like $7 \AA$ suffer from the problem of large scale penetration of the ground state wave function into the barrier region. That defeats the very purpose of a high mobility design. This penetration into the barrier region is reduced substantially, while being conservative about the layer thickness, if we take the thickness as $15 \AA$. We have adopted this thickness for our calculations.

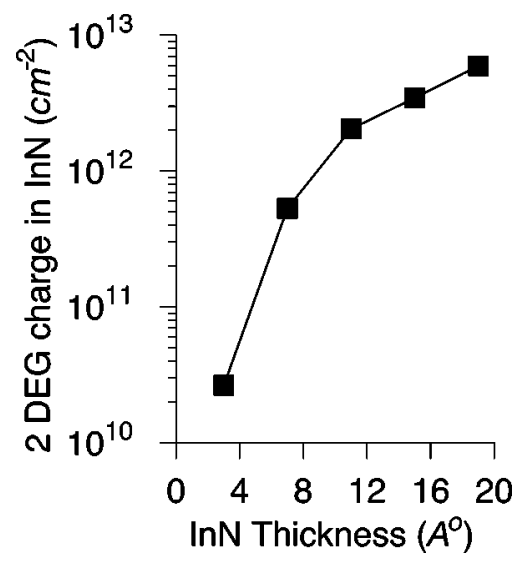

FIG. 4. Charge control study of charge distribution in the $\mathrm{InN}$ layer in the GaN/InN/Al ${ }_{0.37} \mathrm{Ga}_{0.63} \mathrm{~N}$ structure. Thickness of the AlGaN layer is $80 \AA$. Note how the charge in the layer increases as the thickness of the InN layer is increased.

\section{B. Parallel transport calculation}

In the three structures simulated, we find

(1) $\mathrm{GaN} / \mathrm{Al}_{0.37} \mathrm{Ga}_{0.63} \mathrm{~N}(80 \AA)$. This case is included to facilitate comparison with the current generation of HEMTs. In Fig. 5, we present the low and high field velocity-field relationships for this structure, for $L=5 \AA$ and $\Delta=5 \AA$ (in-
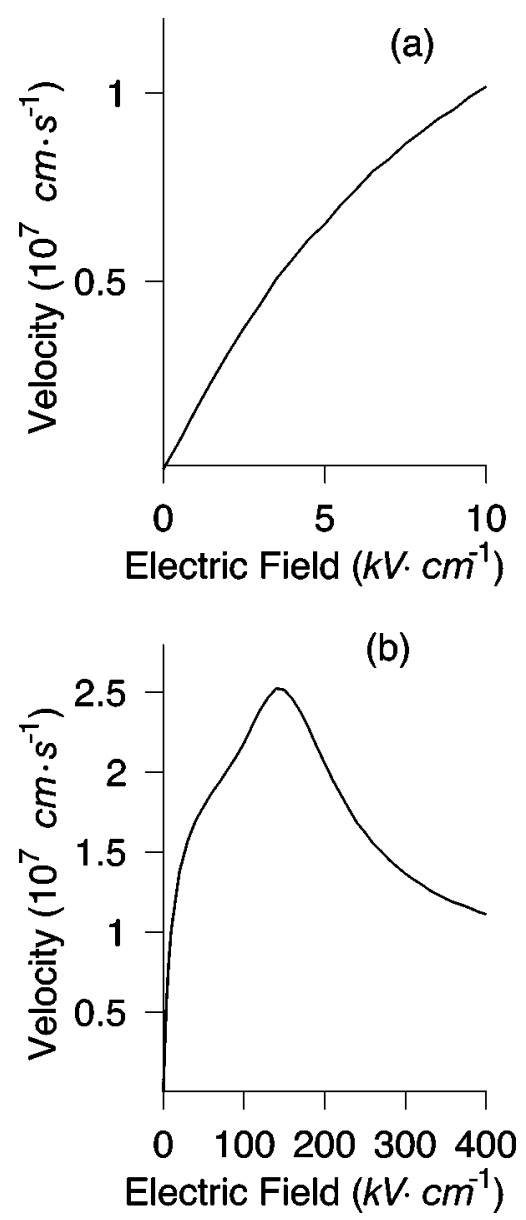

FIG. 5. Monte Carlo study of electron transport in the $\mathrm{GaN} / \mathrm{Al}_{0.37} \mathrm{Ga}_{0.63} \mathrm{~N}$ structure. Thickness of the AlGaN layer is $80 \AA$. (a) Low-field velocity field curves. (b) High-field velocity field curves. 

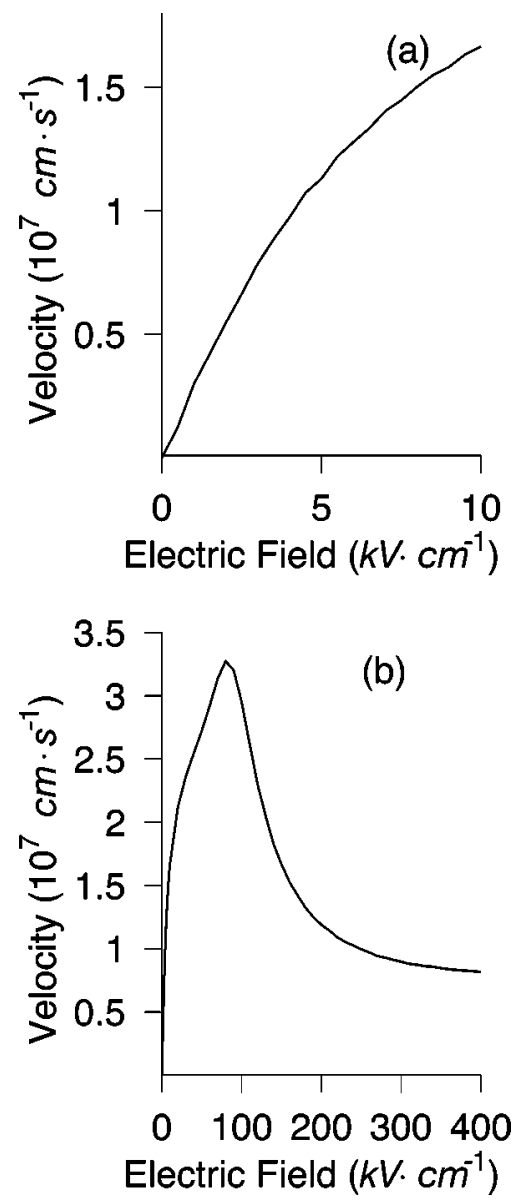

FIG. 6. Results of Monte Carlo simulation with interface roughness parameters $L=5 \AA$ and $\Delta=5 \AA$, with alloy scattering parameter $R=5 \AA$. (a) Low-field velocity field curves. (b) High-field velocity field curves. Note that the peak velocities in this case are considerably higher when compared to Fig. 4.

terface roughness parameters, see Appendix A) and $R=5 \AA$ (alloy scattering parameter, see Appendix B). Low field mobility turns out to be $1490 \mathrm{~cm}^{2}(\mathrm{~V} \mathrm{~s})^{-1}$. This value is reasonable if one considers values reported in literature for barrier layers with lower $\mathrm{Al}$ content. ${ }^{32}$

The results for $\mathrm{GaN}$ channels are similar to the values calculated previously. ${ }^{10}$ An important point to note is that the peak velocity is achieved at rather high fields. Due to the large carrier mass (and high density of states), a very large field is required to bring the carriers to high velocities. It may be noted that for GaAs, the peak velocity occurs at $\sim 3.5$ $\mathrm{kV} / \mathrm{cm}$.

(2) $\mathrm{GaN} / \mathrm{InN}(15 \AA) / \mathrm{Al}_{0.37} \mathrm{Ga}_{0.63} \mathrm{~N}(80 \AA)$. We present velocity field relations for this case in Fig. 6 for $L=5 \AA$, $\Delta=5 \AA$, and $R=5 \AA$. Low field mobility is about $2500 \mathrm{~cm}^{2}$ $(\mathrm{V} \mathrm{s})^{-1}$. This is much higher than the corresponding figure in a conventional GaN/AlGaN HEMT, with the same barrier thickness and $\mathrm{Al}$ composition. This higher mobility arises largely from the confinement of the ground state wave function in the InN channel region. Further, the peak velocity, $v_{\mathrm{pk}}$ is also higher than the corresponding value in the first case.

Early work in the field ${ }^{33}$ suggests a very high value of mobility in $\operatorname{InN}\left[\geqslant 10^{3} \mathrm{~cm}^{2} /(\mathrm{V} \mathrm{s})\right]$. This value is close to the value obtained with interface roughness of $L=5 \AA$ and $\Delta$
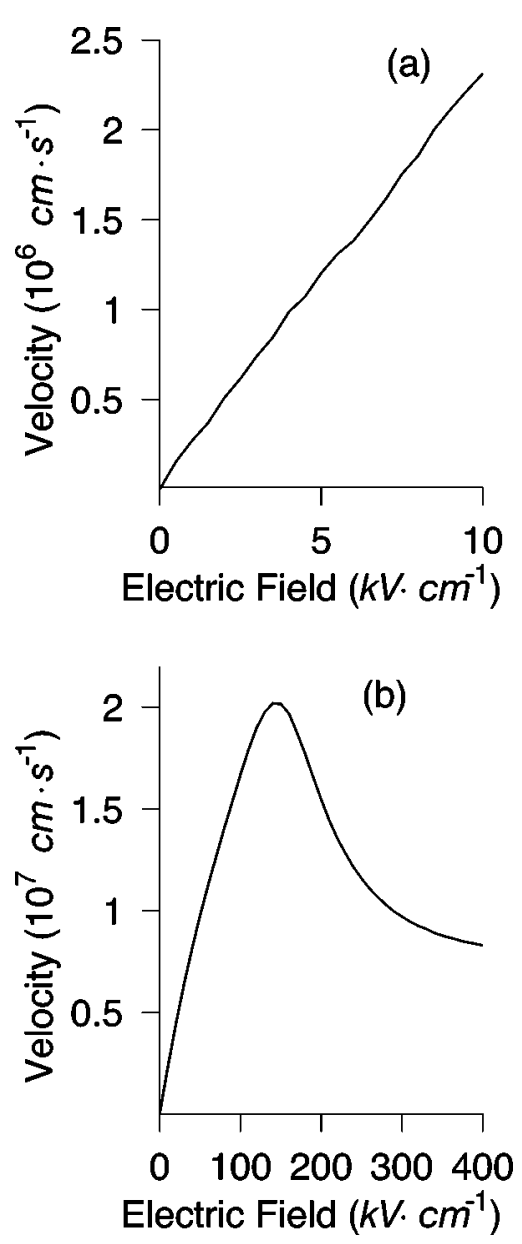

FIG. 7. Results of Monte Carlo simulation with interface roughness parameters $L=70 \AA$ and $\Delta=5 \AA$, with alloy scattering parameter $R=5 \AA$. (a) Low-field velocity field curves. (b) High-field velocity field curves. The low field mobility is much lower than in Fig. 6.

$=5 \AA$. However, it must be understood that this simulation takes two interfaces into account: GaN/InN and InN/Al ${ }_{0.37} \mathrm{Ga}_{0.63} \mathrm{~N}$. Thus it is probable that the interface scattering rate is higher than it would be for a single interface with equivalent roughness, especially due to stronger overlap of the wave function with the interface roughness, owing to strong carrier confinement. Recent experimental studies indicate that the measured Hall mobility in $\mathrm{InN}$ grown on sapphire $^{34}$ is quite low $\left[\sim 300 \mathrm{~cm}^{2}(\mathrm{~V} \mathrm{~s})^{-1}\right]$. If we use a higher value of island length $(L=70 \AA)$ as in Fig. 7, we get a value of $\simeq 307 \mathrm{~cm}^{2}(\mathrm{~V} \mathrm{~s})^{-1}$. The dominant scattering mechanism is polar optical phonon scattering (see Fig. 2). Taking the Hall factor into account, ${ }^{35,36}$ we get a predicted Hall mobility of $\simeq 339 \mathrm{~cm}^{2}(\mathrm{~V} \mathrm{~s})^{-1}$. This is in excellent agreement with the above experimental result.

Other studies ${ }^{37}$ report an even lower value of mobility $\left[\sim 100 \mathrm{~cm}^{2}(\mathrm{~V} \mathrm{~s})^{-1}\right]$. This could be attributed to the poor quality of the interface (indicating a high value of $L$ and $\Delta$ in our model). A recent report of fairly high mobility ${ }^{38}$ for a much thicker structure (InN thickness $\sim 100-800 \AA$ ) is probably due to a lower overlap of the carrier wave function with the interface roughness. No experimental studies for the exact structure being proposed here exist yet. 


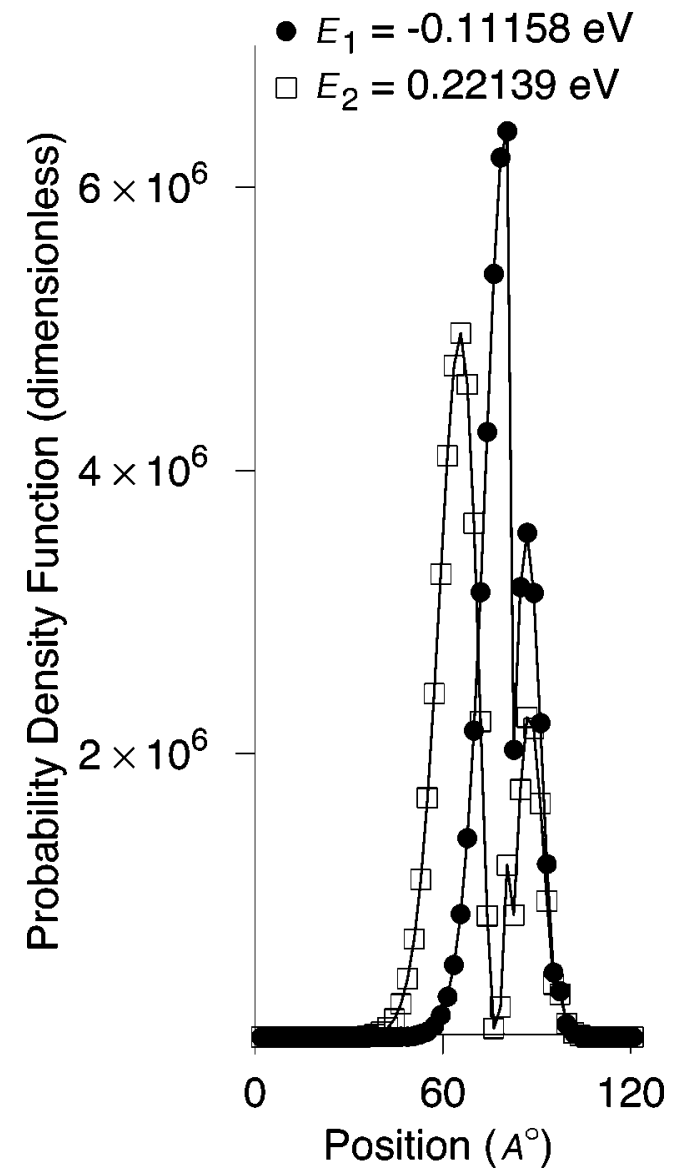

FIG. 8. Probability density functions for the structure, $\mathrm{GaN} / \mathrm{InN} / \mathrm{Al}_{0.37} \mathrm{Ga}_{0.63} \mathrm{~N}$, obtained by solving the charge control problem explained in Sec. II A. The wave functions are normalized over the depth of the device (taken as $300 \AA$ ). The two lowest energy states are shown. The ground state wave function is localized in the $\mathrm{InN}$ region.

It is worthwhile at this point to consider the possibility of breakdown in the low band gap material, InN. In Fig. 8 we show the probability density functions (PDFs) for the two subbands in the $\Gamma$ valley. As can be seen, the ground state wave function, which lies predominantly in the channel region (from 80 to $95 \AA$, an extent of $\sim 15 \AA$ ), is highly localized. That implies that an overlap between this wave function and the wave function of a high energy breakdown initiating state [highly delocalized, as in Fig. 1], would be exceedingly small. According to the discussion in Sec. II B, this implies a very small scattering rate between the two states.

This would reduce the probability of confined state breakdown. However, the standard conduction to valence band breakdown would also be suppressed as the final states in that case are delocalized three-dimensional states.

Due to the very small overlap, our conclusions on breakdown in the GaN/InN channel are:

(a) The threshold energy for breakdown is small $(\sim 0.9$ $\mathrm{eV})$.

(b) However, due to the very small overlap of the high energy state and the band edge state, the breakdown rates are suppressed by a factor of 100 . As a result, the

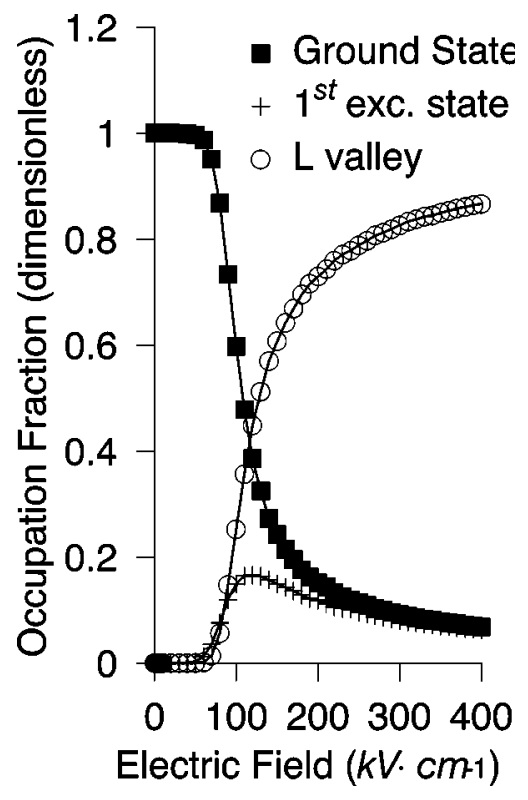

FIG. 9. Wave function occupation fractions for the structure, $\mathrm{GaN} / \mathrm{InN} / \mathrm{Al}_{0.37} \mathrm{Ga}_{0.63} \mathrm{~N}$. It is easy to see wave function switching. Electron occupation in the $\mathrm{X}$ valley is extremely low, and has been omitted for clarity.

breakdown rates are $\sim 10^{11} \mathrm{~s}^{-1}$ and reach values of $\sim 10^{13} \mathrm{~s}^{-1}$ only when electron energies reach $\mathrm{GaN}$ band gap energies.

As will be seen later in this section, the transit time of the carrier across the channel is reduced in structures incorporating InN. That means that for the small residual electron population that might be found in the ground state at high electric fields, the probability of its quick removal from the region is also enhanced, thereby reducing the chance of scattering into a breakdown state even further.

It can be seen from Fig. 9 that most of the electron occupation is associated with the ground state wave function, over a fairly wide range of electric fields. This implies that transport properties are dominated by electrons in the ground state from low fields to moderately high fields. It also may be noted that the electric field at which the occupation fraction in the ground state starts decaying in Fig. 9 also corresponds to the electric field at which electron velocity begins to exhibit a decrease (in Fig. 6).

From the results above, it is easy to discern a trend in the electric field at peak velocity, $E_{\mathrm{pk}}$. Not only is the velocity of the carrier higher for the structures incorporating $\mathrm{InN}$, the peak also occurs at lower electric fields (for comparable values of interface roughness parameters). This means that the carrier would exhibit a peak velocity at much lower electric fields, thereby reducing the transit time across the channel.

To summarize, we present the results of the calculation in Table II. It may be noted that the simulated electron mobilities are the highest for the second structure, $\mathrm{GaN} / \mathrm{InN}(15 \AA) / \mathrm{Al}_{0.37} \mathrm{Ga}_{0.63} \mathrm{~N}(80 \AA)$, with $L=5 \AA, \quad \Delta$ $=5 \AA$, and $R=5 \AA$. It is also worth noting that the mobilitycharge product, which quantifies the current carrying capa- 
TABLE II. Summary of results of calculations: peak velocity, $v_{\mathrm{pk}}$, electric field at peak velocity, $E_{\mathrm{pk}}$, low-field drift mobility, $\mu_{l}, 2$ DEG charge in channel region (InN in case of structures II and III), $n_{2 \text { DEG }}$, and the mobility-charge product. The thickness of the $\mathrm{AlGaN}$ layer is $80 \AA$, and the thickness of the InN channel is 15 $\AA$. Structure I: GaN/ $/ \mathrm{Al}_{0.37} \mathrm{Ga}_{0.63} \mathrm{~N}$, II: $\mathrm{GaN} / \mathrm{InN} / \mathrm{Al}_{0.37} \mathrm{Ga}_{0.63} \mathrm{~N}$, interface island length $=5 \AA$ and III: $\mathrm{GaN} / \mathrm{InN} / \mathrm{Al}_{0.37} \mathrm{Ga}_{0.63} \mathrm{~N}$, interface island length $=70 \AA$.

\begin{tabular}{lcclcc}
\hline \hline Structure & $v_{\mathrm{pk}}\left(\mathrm{cm} \mathrm{s}^{-1}\right)$ & $E_{\mathrm{pk}}\left(\mathrm{V} \mathrm{cm}^{-1}\right)$ & $\mu_{l}\left(\mathrm{~cm}^{2} \mathrm{~V}^{-1} \mathrm{~s}^{-1}\right)$ & $n_{2 \text { DEG }}\left(\mathrm{cm}^{-2}\right)$ & $\mu_{l} \cdot n\left(\mathrm{~V}^{-1} \mathrm{~s}^{-1}\right)$ \\
\hline I & $2.52 \times 10^{7}$ & $1.40 \times 10^{5}$ & $\approx 1490$ & $1.13 \times 10^{13}$ & $1.69 \times 10^{16}$ \\
II & $3.27 \times 10^{7}$ & $8.00 \times 10^{4}$ & $\approx 2501$ & $3.46 \times 10^{12}$ & $8.64 \times 10^{15}$ \\
III & $2.02 \times 10^{7}$ & $1.50 \times 10^{5}$ & $\approx 307$ & $3.46 \times 10^{12}$ & $1.06 \times 10^{15}$ \\
\hline \hline
\end{tabular}

bility of the device, is not too adversely affected by the introduction of the InN layer (see Table II).

\section{CONCLUSIONS}

In this work we have presented two sets of results, one for $\mathrm{GaN} / \mathrm{AlGaN}$ and a set for different values of interface roughness parameters in $\mathrm{GaN} / \mathrm{InN} / \mathrm{AlGaN}$. The latter structure has been designed with the twin constraints of strain energy and available charge, for electron population redistribution at high fields. The effect of such wave function switching is manifest-the high mobility capability of the InN channel is exploited at relatively low electric fields, while high breakdown capability of the AlGaN and $\mathrm{GaN}$ layers is utilized at relatively high electric fields. The probability of a breakdown occurring in the low band gap channel region is reduced by the combined effects of low degree of overlap between the bound states in the channel and the breakdown delocalized states, and the fast transit times of the carriers in the channel at lower electric fields.

As growth technology matures, it is possible in principle to incorporate even thicker layers of $\mathrm{InN}$, which would increase the amount of charge in the 2DEG (see Fig. 3). This would increase the mobility-charge product (see Table II). One of the immediately apparent advantages of this scheme is that the electric field at peak velocity, $E_{\mathrm{pk}}$, can be tailored by varying the thickness of the channel region, quality of the interface, as well as the material (for instance, using $\mathrm{In}_{x} \mathrm{Ga}_{1-x} \mathrm{~N}$ instead of pure InN). Thus from a device designer's standpoint, this structure is capable of more flexibility.

Recent work ${ }^{39}$ on $\operatorname{In}_{x} \mathrm{Ga}_{1-x} \mathrm{~N} / \mathrm{GaN}$ quantum wells indicate that it is possible to grow extremely smooth surfaces for $x>0.3$. Future work would involve an investigation of the effect of using $\operatorname{In}_{x} \mathrm{Ga}_{1-x} \mathrm{~N}$ layers in the channel region (with $x<1$ ) to make the use of thicker channel regions possible.

\section{ACKNOWLEDGMENTS}

This work was supported by Grants No. F001681 (the POLARIS program) and No. F004815 from the U.S. Office of Naval Research.

\section{APPENDIX A: INTERFACE ROUGHNESS SCATTERING}

Though prior calculations exist in literature for this mechanism, ${ }^{21}$ we repeat the calculation since we consider bandwise averaging of material parameters (see Sec. II D) in the 2DEG system. Consider the situation in Fig. 3(a). We model interface roughness as a disturbance in the potential at the interface of two materials, thereby forming islands. The parameter $y$ quantifies the spacing of these islands. We define the overlap integral between two envelope states as

$$
I_{i j}(\Delta) \equiv\langle j \mid i\rangle=\int_{-\Delta / 2}^{\Delta / 2} \psi_{j}^{*}(z) \psi_{i}(z) d z
$$

The $\psi$ functions above are normalized envelope functions in single dimension. Total wave function is

$$
\begin{aligned}
& \psi_{i, \mathbf{k}}^{\sim}(\mathbf{r})=\frac{e^{i \rho \cdot \mathbf{k}_{\|}}}{\sqrt{A}} \psi_{i}(z), \\
& \text { and } \psi_{i, \mathbf{k}}^{*}(\mathbf{r})=\frac{e^{-i \rho \cdot \mathbf{k}_{\|}}}{\sqrt{A}} \psi_{i}^{*}(z),
\end{aligned}
$$

where $A$ is an arbitrary normalization area. The Hamiltonian for the interaction can be written as

$$
H^{\prime}=\left\{\begin{array}{cc}
U_{0} & \text { if } \quad|\rho|<\frac{L}{2} ;|z|<\frac{\Delta}{2} \\
0 & \text { otherwise, }
\end{array}\right.
$$

where $U_{0}$ is the potential difference that the electrons experience between one island and the other. At this stage of the calculation, $L$ stands for a general length of the island, not the length $L$ indicated in Fig. 2. The matrix element of the interaction can be written as

$$
\begin{aligned}
\underbrace{\left\langle i, \mathbf{k}\left|H^{\prime}\right| j, \mathbf{k}^{\prime}\right\rangle}_{M_{i j}\left(\mathbf{k}, \mathbf{k}^{\prime}\right)} & =\frac{U_{0, j}}{A} \int_{\text {Area }} d A e^{\imath \rho \cdot\left(\mathbf{k}_{\mid \ell}-\mathbf{k}_{||}^{\prime}\right)} \underbrace{I_{i j}(\Delta)}_{E q \cdot(A 1)} \\
& =\frac{U_{0, j} I_{i j}(\Delta)}{A} \int_{0}^{\frac{L_{j}}{2}} \rho d \rho \int_{0}^{2 \pi} d \theta e^{\imath \rho q_{i j} \cos \theta} \\
& =\frac{\pi I_{i j}(\Delta) U_{0, j} L_{j}}{A q_{i j}} J_{1}\left(\frac{q_{i j} L_{j}}{2}\right)
\end{aligned}
$$

where $q_{i j}=\left|\mathbf{k}^{\prime}-\mathbf{k}\right|$. The reason for the extra subscripts on $U_{0}$ and $L$ is that they are averaged over each of the subbands (see Sec. II D). This is necessary because the spatial variation of each subband is unique. The scattering rate between states $|i, \mathbf{k}\rangle$ and $\left|j, \mathbf{k}^{\prime}\right\rangle$ can be written as 


$$
\begin{aligned}
W_{i j}\left(\mathbf{k}, \mathbf{k}^{\prime}\right) \equiv & \frac{2 \pi}{\hbar}\left|M_{i j}\left(\mathbf{k}, \mathbf{k}^{\prime}\right)\right|^{2} \delta\left(E_{j, \mathbf{k}^{\prime}}-E_{i, \mathbf{k}}\right) \\
= & \frac{2 \pi}{\hbar} \frac{\pi^{2} I_{i j}^{2}(\Delta) U_{0, j}^{2} L_{j}^{2}}{A^{2} q_{i j}^{2}} J_{1}^{2}\left(\frac{q_{i j} L}{2}\right) \\
& \times \delta\left(\frac{\hbar^{2}}{2 m}\left(k^{\prime 2}-k^{2}\right)+\Delta E_{i j}\right) .
\end{aligned}
$$

This can be simplified using the definition, $k_{i j}$ $\equiv \sqrt{2 m \Delta E_{i j} / \hbar^{2}}$. Using the properties of the Dirac delta function, we get

$$
\begin{aligned}
W_{i j}\left(\mathbf{k}, \mathbf{k}^{\prime}\right)= & \frac{4 \pi^{3} m_{j} I_{i j}^{2}(\Delta) U_{0, j}^{2} L_{j}^{2} J_{1}^{2}\left(\frac{q_{i j} L}{2}\right)}{A^{2} q_{i j}^{2} \hbar^{3}} \\
& \times \delta\left[k^{\prime 2}-\left(k^{2}-k_{i j}^{2}\right)\right] .
\end{aligned}
$$

The total scattering rate is given by splitting up the volume integral into an areal integral and a discrete sum in the confinement direction, over subbands.

Using Eq. (A4), we get

$$
\begin{aligned}
W_{i}(\mathbf{k})= & \frac{1}{2 A \hbar^{3}} \sum_{j=1}^{n} m_{j} U_{0, j}^{2} L_{j}^{2} I_{i j}^{2}(\Delta) \int_{0}^{2 \pi} d \theta \int_{0}^{\infty} k^{\prime} d k^{\prime} \\
& \times \frac{J_{1}^{2}\left(\frac{q_{i j} L_{j}}{2}\right)}{q_{i j}^{2}}(1-\cos \theta) \delta\left(k^{\prime 2}-k^{2}+k_{i j}^{2}\right) .
\end{aligned}
$$

This expression is quite complicated in general, but can be simplified considerably if we neglect all intersubband transitions (i.e., consider the scattering to be completely elastic). Thus $k_{i j} \equiv 0, q_{j j}^{2} \equiv 4 k_{j}^{2} \sin ^{2} \theta / 2$, and we can write

$$
W_{i}(\mathbf{k})=\frac{m_{i} U_{0, i}^{2} L_{i}^{2} I_{i i}^{2}(\Delta)}{4 A \hbar^{3} k_{i}^{2}} \int_{0}^{\pi} J_{1}^{2}\left(k_{i} L_{i} \sin \phi\right) d \phi .
$$

The scattering rate from one island of length $y L_{i}$ is

$$
\begin{aligned}
W_{i}(\mathbf{k})= & \frac{m_{i}(1-y)^{2} U_{0, i}^{2}\left(y L_{i}\right)^{2} I_{i i}^{2}(\Delta)}{4 A \hbar^{3} k_{i}^{2}} \\
& \times \int_{0}^{\pi} J_{1}^{2}\left(k_{i} y L_{i} \sin \phi\right) d \phi .
\end{aligned}
$$

The corresponding scattering rate from the island of length $(1-y) L_{i}$ is

$$
\begin{aligned}
W_{i}(\mathbf{k})= & \frac{m_{i} y^{2} U_{0, i}^{2}\left[(1-y) L_{i}\right]^{2} I_{i i}^{2}(\Delta)}{4 A \hbar^{3} k_{i}^{2}} \\
& \times \int_{0}^{\pi} J_{1}^{2}\left[k_{i}(1-y) L_{i} \sin \phi\right] d \phi .
\end{aligned}
$$

There are $4 A y / \pi\left(y L_{i}\right)^{2}$ islands of length $y L_{i}$ and $4 A(1-y) / \pi\left[(1-y) L_{i}\right]^{2}$ of length $(1-y) L_{i}$. Taking this into account, the total scattering rate is given by

$$
\begin{aligned}
W_{i}(\mathbf{k})= & \frac{m_{i}(1-y) y U_{0, i}^{2} I_{i i}^{2}(\Delta)}{\pi \hbar^{3} k_{i}^{2}} \\
& \times\left[(1-y) \int_{0}^{\pi} J_{1}^{2}\left(k_{i} y L_{i} \sin \phi\right) d \phi\right. \\
& \left.+y \int_{0}^{\pi} J_{1}^{2}\left[k_{i}(1-y) L_{i} \sin \phi\right] d \phi\right] .
\end{aligned}
$$

\section{APPENDIX B: ALLOY SCATTERING}

We divide the volume of the alloy into separate scattering layers, each consisting of an array of scattering centers. Each scattering center has the shape indicated in Fig. 3(b). The choice of this hard cylinder model, instead of the more usual hard sphere model, is suggested by the underlying geometry of the decomposition. Electron states are quantized in one spatial direction while maintaining their free particle nature in the other directions. That breaks the directional degeneracy found in more conventional treatments of the problem. The overlap integral at each scattering center (positioned at $z_{0}$ ) can be written as

$$
I_{i j}\left(z_{0}, R\right)=\left\langle\psi_{j} \mid \psi_{i}\right\rangle=\int_{z_{0}-R / 2}^{z_{0}+R / 2} \psi_{j}^{*}(z) \psi_{i}(z) d z
$$

The Hamiltonian for the interaction can be written as

$$
H^{\prime}=\left\{\begin{aligned}
U_{0} & \text { if }|\rho|<\frac{R}{2} ;|z|<R \\
& 0 \text { otherwise. }
\end{aligned}\right.
$$

As might be expected, the calculation of scattering rate from one scattering layer proceeds with steps identical to those discussed in Appendix A. Ignoring intersubband scattering as before, we get,

$$
W_{i}(\mathbf{k})=\frac{m_{i} I_{i i}^{2}\left(z_{0}, R_{i}\right) R_{i}^{2} U_{0, i}^{2}}{4 A \hbar^{3} k^{2}} \int_{0}^{\pi} J_{1}^{2}\left(k R_{i} \sin \phi\right) d \phi .
$$

This is the scattering rate from one island. In each such layer, there are $4 A(1-x) / \pi R^{2}$ atoms of type $\mathrm{I}$ and $4 A x / \pi R^{2}$ atoms of type II. Summing these (along with the correct potential in each case), we get the total scattering rate from one scattering layer as

$$
\begin{aligned}
W_{i}(\mathbf{k})= & \frac{m_{i} I_{i i}^{2}\left(z_{0}, R_{i}\right) x(1-x) U_{0, i}^{2}}{\pi \hbar^{3} k^{2}} \\
& \times \int_{0}^{\pi} J_{1}^{2}\left(k R_{i} \sin \phi\right) d \phi .
\end{aligned}
$$

To find the total scattering rate, we need to sum over all the layers. There are $L_{a l} / R$ such layers $\left(L_{a l}\right.$ is the thickness of the alloy layer). Thus

$$
\begin{aligned}
W_{i}(\mathbf{k})= & \frac{m_{i} U_{0, i}^{2} x(1-x)}{\pi \hbar^{3} k^{2}} \sum_{p=1}^{p=L_{a l} / R_{i}} I_{i i}^{2}\left(p \frac{R_{i}}{2}, R_{i}\right) \\
& \times \int_{0}^{\pi} J_{1}^{2}\left(k R_{i} \sin \phi\right) d \phi .
\end{aligned}
$$


At first sight, it might appear that the above sum adduces a geometrical structure composed of cylindrical columns that run through the material, instead of an uncorrelated pattern of scattering centers. However, the averaging in each layer consisted of randomly placed scattering centers. Thus there would be no such columns and the alloy would be random, as it ought to be, by definition.

${ }^{1}$ V. Y. Davydov, A. A. Klochikhin, R. P. Seisyan, V. V. Emstev, S. V. Ivanov, F. Bechstedt, J. Furthmüller, H. Harima, A. V. Mudryi, J. Aderhold, O. Smechinova, and J. Graul, Phys. Status Solidi B 229, R1 (2002).

${ }^{2}$ V. Y. Davydov, A. A. Klochikhin, V. V. Emtsev, S. V. Ivanov, V. V. Vekshin, F. Bechstedt, J. Furthmüller, H. Harima, A. V. Murdyi, A. Hashimoto, A. Yamamoto, J. Aderhold, J. Graul, and E. E. Haller, Phys. Status Solidi B 230, R4 (2002).

${ }^{3}$ J. Wu, W. Walukiewicz, K. M. Yu, J. W. A. III, E. E. Haller, H. Lu, W. J. Schaff, Y. Saito, and Y. Nanishi, Appl. Phys. Lett. 80, 3967 (2002).

${ }^{4}$ T. L. Tansley and C. P. Foley, J. Appl. Phys. 59, 3241 (1986).

${ }^{5}$ S. K. O'Leary, B. E. Foutz, M. S. Shur, U. V. Bhapkar, and L. F. Eastman, J. Appl. Phys. 83, 826 (1998).

${ }^{6}$ E. O. Kane, J. Phys. Chem. Solids 8, 38 (1959).

${ }^{7}$ D. A. Broido and L. J. Sham, Phys. Rev. B 31, 888 (1985).

${ }^{8} \mathrm{~J}$. Singh, Physics of Semiconductors and Their Heterostructures (McGraw-Hill, New York, 1993).

${ }^{9}$ B. E. Foutz, S. K. O’Leary, M. S. Shur, and L. F. Eastman, J. Appl. Phys. 85, 7727 (1999).

${ }^{10}$ Y. Zhang and J. Singh, J. Appl. Phys. 89, 386 (2001).

${ }^{11}$ F. Bernardini, V. Fiorentini, and D. Vanderbilt, Phys. Rev. B 56, 10024 (1997).

${ }^{12}$ O. Ambacher, J. Smart, J. R. Shealy, N. G. Weimann, K. Chu, M. Murphy, W. J. Schaff, L. F. Eastman, R. Dimitrov, L. Wittmer, M. Stutzmann, W. Rieger, and J. Hilsenbeck, J. Appl. Phys. 85, 3222 (1999).

${ }^{13}$ Y. Zhang and J. Singh, J. Appl. Phys. 85, 587 (1999).

${ }^{14}$ M. Singh, Y. Zhang, J. Singh, and U. Mishra, Appl. Phys. Lett. 77, 1867 (2000).

${ }^{15}$ K. Yokoyama and K. Hess, Phys. Rev. B 33, 5595 (1986).

${ }^{16}$ R. F. Davis, A. M. Roskowski, E. A. Preble, J. S. Speck, B. Heying, J. A. Freitas, Jr., E. R. Glaser, and W. E. Carlos, Proc. IEEE 90, 993 (2002).

${ }^{17}$ P. J. Price, Ann. Phys. (N.Y.) 133, 217 (1981).

${ }^{18}$ F. A. Riddoch and B. K. Ridley, J. Phys. C 16, 6971 (1983).

${ }^{19}$ L. I. Schiff, Quantum Mechanics (McGraw-Hill, New York, 1968).
${ }^{20}$ F. Schwabl, Quantum Mechanics (Springer-Verlag, Berlin, 1991).

${ }^{21}$ W.-P. Hong, J. Singh, and P. K. Bhattacharya, IEEE Electron Device Lett. EDL-7, 480 (1986).

${ }^{22}$ M. Farahmand, C. Garetto, E. Bellotti, K. J. Brennan, M. Goano, E. Ghilino, G. Ghione, J. D. Albrecht, and P. P. Ruden, IEEE Trans. Electron Devices 48, 535 (2001).

${ }^{23}$ V. W. L. Chin, T. L. Tansley, and T. Osotchan, J. Appl. Phys. 75, 7365 (1994)

${ }^{24}$ A. S. Barker, Jr. and M. Ilegems, Phys. Rev. B 7, 743 (1973).

${ }^{25}$ R. R. Reeber and K. Wang, MRS Internet J. Nitride Semicond. Res. 6, 1 (2001)

${ }^{26}$ V. Y. Davydov, A. A. Klochikhin, M. B. Smirnov, V. V. Emtsev, V. D. Petrikov, I. A. Abroyan, A. I. Titov, I. N. Goncharuk, A. N. Smirnov, V. V. Mamutin, S. V. Ivanov, and T. Inushima, Phys. Status Solidi B 216, 779 (1999).

${ }^{27}$ V. Y. Davydov, Y. E. Kitaev, I. N. Goncharuk, A. M. Tsaregorodtsev, A. N. Smirnov, A. O. Lebedev, V. M. Botnaryk, Y. V. Zhilyaev, M. B. Smirnov, A. P. Mirgorodsky, and O. K. Semchinova, J. Cryst. Growth 189/190, 656 (1998).

${ }^{28}$ I. Vurgaftman, J. R. Meyer, and L. R. Ram-Mohan, J. Appl. Phys. 89, 5815 (2001).

${ }^{29}$ J. A. Majewski, G. Zandler, and P. Vogl, J. Phys.: Condens. Matter 14, 3511 (2002)

${ }^{30}$ A. T. Meney, E. P. O'Reilly, and A. R. Adams, Semicond. Sci. Technol. 11, 897 (1996).

${ }^{31}$ S. Keller, I. Ben-Yacov, S. P. DenBaars, and U. Mishra (private communication).

${ }^{32}$ I. P. Smorchkova, C. R. Elsass, J. P. Ibbetson, R. Ventury, B. Heying, P. Fini, E. Haus, S. P. DenBaars, J. S. Speck, and U. K. Mishra, J. Appl. Phys. 86, 4520 (1999).

${ }^{33}$ T. L. Tansley and C. P. Foley, Electron. Lett. 20, 1066 (1984).

${ }^{34}$ A. Yamamoto, T. Shin-ya, T. Sugiura, and A. Hashimoto, J. Cryst. Growth 189/190, 461 (1998).

${ }^{35}$ S. M. Sze, Physics of Semiconductor Devices, 2nd ed. (Wiley, New York, 1981).

${ }^{36}$ K. Seeger, Semiconductor Physics-An Introduction (Springer-Verlag, Berlin, 1999).

${ }^{37}$ T. Beierlein, S. Strite, A. Dommann, and D. J. Smith, MRS Internet J. Nitride Semicond. Res. 2, 27 (1997).

${ }^{38}$ H. Lu, W. J. Schaff, L. F. Eastman, and C. Wood, Mater. Res. Soc. Symp. Proc. 693, I1.5.1 (2002)

${ }^{39}$ M. G. Choeng, S. M. Jeong, H. S. Yoon, C. S. Kim, R. J. Choi, E. J. Hwang, E.-K. Suh, and H. J. Lee, J. Korean Phys. Soc. 40, 300 (2002). 